\title{
BIO_13 - Interaction study of CD20 and Rituximab's scFv to propose an anti-CD20 CAR
}

Alison de Sousa Rebouças ${ }^{1 *}$; Marcos Roberto Lourenzoni².

${ }^{1}$ UFCe - Universidade Federal do Ceará;

${ }^{2}$ Fiocruz Ceará.

Introduction: CD20 is an integral protein of B lymphocytes, being a marker of tumor cells such as nonHodgkin's lymphoma and target of immunotherapies like Chimeric Antigenic Receptors in T cells (CAR-T Cell). CAR-T Cell is directed to cancer cells due to its specificity to the CD20 antigen, improving function in passive immunotherapy. CD20 structure was obtained in 2020 by cryoelectromicroscopy and deposited at PDB 6VJA. The development of anti-CD20 monoclonal antibodies (mAbs), such as Rituximab, has always been carried out with extracellular CD20 fragments. From the complete structure, more elaborate studies of Molecular Dynamics (MD) simulation can access structural and energetic information that are related to the affinity between CD20 and the antibody single chain fragment variable (scFv). This study will allow a better understanding of the interaction mechanism to propose a scFv model more appropriate to the CAR construction.

Objective: To study the interaction between Rituximab's scFv and CD20 with its 3D structure immersed in a membrane model, in order to find information that explains the high affinity of Rituximab for CD20 and use it in the design of a new scFv to be part of CAR.

Methodology: The secondary structure of $\mathrm{scFV}$ was obtained from $\mathrm{V}_{\mathrm{H}}$ and $\mathrm{V}_{\mathrm{L}}$ coordinates of PDB 6VJA and a linker $\left[\mathrm{G}_{4} \mathrm{~S}\right]_{3}$. The CD20 dimer and the dimer-2scFv complex were inserted in a POPC membrane model, obtained from CHARMM-GUI. The two systems were submitted to structural equilibrium phase using the CHARMM36m force field using Gromacs 2018. The CD20 dimer DM followed for 500ns, while the complex was simulated in triplicate $(\mathrm{n} 1, \mathrm{n} 2$ and $\mathrm{n} 3)$ for $600 \mathrm{~ns}$, starting from different initial velocities. The structural maintenance of both systems was evaluated by calculating RMSD and clustering analysis (gmx_rms and gmx_cluster programs of Gromacs package). The intermolecular interaction potential (IIP), the sum of Lennard-Jones and Coulomb potentials, between the scFv and CD20 residues were measured along the simulations by a homemade program.

Results: RMSD calculated for the CD20 dimer was $0.32 \pm 0.03 \mathrm{~nm}$ after the equilibrium time $\left(\mathrm{t}_{\mathrm{eq}}\right)$ of $220 \mathrm{~ns}$. While for the dimer-2scFv complex, RMSD was $0.40 \pm 0.08 \mathrm{~nm}$ after $\mathrm{t}_{\mathrm{eq}}$ of $360 \mathrm{~ns}$, also showing maintenance of the structure in a POPC environment interfaced by water. The scFv residues that present IIP most attractive to CD20 are D56 and K64 from CDR H2 and N228 from CDR L3.

Conclusion: The scFv-CD20 interaction analysis allowed us to identify that residues D56, K64 and N228 are key to explain the high affinity of Rituximab for CD20. From the understanding of the interaction mechanism, the proposition of antibody variants can be explored to build an anti-CD20 CAR model to act in cell therapy.

Keywords: CAR-T Cell; CD20; Molecular Dynamics 